\section{Fatores associados ao sofrimento psíquico de policiais militares da cidade do Rio de Janeiro, Brasil}

\author{
Factors associated with psychological distress \\ among military police in Rio de Janeiro, Brazil
}

\author{
1 Escola Nacional de Saúde \\ Pública Sergio Arouca, \\ Fundação Oswaldo Cruz, \\ Rio de Janeiro, Brasil.

\section{Correspondência} \\ E. R. Souza \\ Centro Latino-americano de \\ Estudos de Violência e Saúde \\ Jorge Careli, Escola Nacional \\ de Saúde Pública Sergio \\ Arouca, Fundação Oswaldo \\ Cruz. \\ Av. Brasil 4036, sala 700, \\ Rio de Janeiro, $R J$ \\ 21040-361, Brasil. \\ edinilsaramos@gmail.com
}

\section{Abstract}

This study investigates factors associated with psychological distress among military police $(n=1,120)$ in Rio de Janeiro, Brazil. The article describes their social, economic, and demographic characteristics, quality of life, mental health, and work conditions. Measurement of psychological distress used the Self-Reported Questionnaire. Analysis of associations used logistic regression, considering factors associated with psychological distress. The results indicate an association between psychological distress and factors such as ability to react to difficult situations, dissatisfaction with life, health problems (especially digestive, nervous, and musculoskeletal symptoms), and adverse work conditions such as excessive workload, constant stress, and victimization. The article concludes by highlighting the need for health promotion interventions for the military police, focusing especially on their mental health.

Psychological Stress; Police; Mental Health
Edinilsa Ramos de Souza 1 Maria Cecília de Souza Minayo 1 Juliana Guimarães e Silva 1 Thiago de Oliveira Pires 1

\section{Introdução}

Policiais, em todo o mundo, constituem uma das categorias de trabalhadores com maior risco de vida e de estresse 1,2,3,4,5,6,7,8,9. No caso específico dos policiais militares, o nível de estresse tem sido apontado como superior ao de outras categorias profissionais, não só pela natureza das atividades que realizam, mas também pela sobrecarga de trabalho e pelas relações internas à corporação cuja organização se fundamenta em hierarquia rígida e disciplina militar. Tais características estruturantes tornam a instituição resistente a mudanças e repercutem na saúde física e mental dos servidores. Destacam-se, ainda, como fontes geradoras de estresse, as relações, por vezes, tensas e conflituosas dos policiais com o Sistema de Justiça e com o público a quem atendem 5,6.

No tocante aos policiais militares do Rio de Janeiro, as condições de saúde e de trabalho tendem a ser extremas, pois eles lidam com elevados índices de criminalidade de grupos organizados de criminosos armados 5,6,7. Os constantes riscos a que o policial militar se expõe em função do exercício da sua profissão levam-no, geralmente, a sentir medo, por si mesmo e por sua família, tanto de ser reconhecido como agente da segurança nos períodos de folga do trabalho, quando aumenta seu risco de vitimização, como de ser agredido e morto no desempenho das suas funções 6 . Esse medo é uma forma de defesa do 
corpo e do espírito dos que vivem sempre alerta aos perigos. No entanto, quando o estado de tensão e o desgaste físico e emocional são constantes, eles podem gerar diversos prejuízos à saúde e à qualidade de vida, dentre eles, estresse e sofrimento psíquico.

Ao discutir a organização laboral, a carga mental e o sofrimento psíquico, Wisner 10 afirma que as condições de trabalho estão constituídas pelos componentes físico, cognitivo e psíquico, dos quais, o último é o elemento mais difícil de se caracterizar, pois ele se relaciona ao significado que tais condições têm para cada trabalhador, como também ressaltam Dejours \& Abdoucheli 11. O conceito de sofrimento psíquico é aqui entendido como transtorno psiquiátrico menor ou doença psiquiátrica não psicótica, usado para designar vários tipos de sintomas que traduzem sofrimento ${ }^{6}$. Dejours 12 considera-o como um mal-estar inespecífico, intermediário entre a saúde e a doença, mas que pode se tornar patológico, dependendo da frequência de sua ocorrência. Neste artigo, busca-se investigar fatores associados ao sofrimento psíquico dos policiais militares da cidade do Rio de Janeiro.

\section{Metodologia}

Este trabalho analisa parte dos dados de uma pesquisa de corte transversal cujo objetivo foi estudar a qualidade de vida e as condições de saúde e de trabalho dos policiais militares do Rio de Janeiro ${ }^{6}$. A investigação original, realizada entre 2005 e 2007, foi constituída por abordagens quantitativa e qualitativa. Este texto traz a análise apenas de alguns dados quantitativos.

Foi calculada uma amostra estratificada e por conglomerados em um estágio. Os seis estratos compreendiam a natureza do serviço (administrativo ou operacional) e, em cada um deles, os cargos (oficial, suboficial e não oficial). Considerou-se um conglomerado como a unidade física (batalhão, quartel etc.) em que todos os profissionais ali alocados poderiam ser entrevistados. Para o cálculo, foram incluídas todas as unidades da Polícia Militar localizadas na capital e o efetivo de cada uma delas, segundo os cargos. Foram sorteadas 18 unidades com seus respectivos efetivos. Nelas, foi abordado o montante necessário de policiais de cada cargo definido pelo cálculo amostral, que totalizou 1.700 agentes.

Para uma confiança de 95\% (IC95\%), a partir de uma amostra aleatória simples de 610 pessoas, é possível se obter, para proporções maiores que $40 \%$, um erro de amostragem menor ou igual a 3,5 pontos percentuais quando desprezamos o efeito do coeficiente de correlação in- traclasse. Dependendo da magnitude desse coeficiente, o erro deverá ser maior, caso o mesmo seja positivo.

Os questionários foram distribuídos individualmente, em envelope fechado, tanto diretamente aos policiais como ao responsável pela sua distribuição nas unidades. Desses, 1.120 foram respondidos; 199 foram devolvidos sem preenchimento; e 381 não foram devolvidos. As perdas atingiram $34,1 \%$ e se deveram à recusa dos gestores e policiais em participar e à divergência entre o contingente real e o informado na listagem fornecida pela corporação.

Aplicou-se um questionário anônimo com 107 questões fechadas, mas, neste artigo, foram analisadas 40 questões que, a partir de estudos já realizados $6,7,8$, apresentam relação teórica com o sofrimento psíquico e que compõem quatro blocos.

Bloco 1 - características socioeconômicas e demográficas dos policiais (idade, sexo, situação conjugal, renda etc.); bloco 2 - qualidade de vida (capacidade de reagir a situações difíceis, satisfação com a vida, apoio social, dentre outras); bloco 3 - condições de saúde (prática de atividade física, lesões permanentes causadas pelo trabalho, alterações em órgãos e sistemas, consumo de substâncias etc.) e bloco 4 - condições de trabalho (tempo de serviço na polícia, condição de vida após ingresso na corporação, trabalhar além do horário etc.).

O apoio social foi mensurado pela Escala de Apoio Social 13, composta por 19 itens e cinco dimensões (material, afetiva, interação positiva, emocional e informação) utilizadas como variáveis para se estimar a qualidade de vida.

Para mensurar a existência de sofrimento psíquico ou de distúrbios psiquiátricos menores, aqui entendida como variável dependente, utilizou-se a escala Self-Reported Questionnaire (SRQ-20), desenvolvida por Harding et al. 14, em 1980, e validada, no Brasil, por Mari \&Williams 15. Essa escala possui 20 itens com respostas dicotômicas do tipo sim/não e se refere a transtornos de elevada prevalência em nível populacional que costumam estar relacionados, indiretamente, às condições socioeconômicas e, mais diretamente, aos eventos de vida estressantes 16,17,18. Foram considerados portadores de sofrimento psíquico ou distúrbio psiquiátrico menor os policiais com sete ou mais respostas positivas.

Foi considerado consumo de substância o uso de, pelo menos, um dos seguintes componentes: álcool, cigarro, remédio para emagrecer, maconha, tranquilizante, sedativo, anabolizante, cocaína, entre outros.

$\mathrm{Na}$ análise, em um primeiro momento, foram feitos testes de associação entre a variável 
resposta (ter ou não sofrimento psíquico) e as variáveis independentes; para isso, foi utilizado o F ajustado, uma versão do teste de Rao-Scott 19 . Variáveis com valor de $\mathrm{p}<0,05$ foram incluídas no modelo.

Foi empregado o modelo com enfoque hierarquizado 20 , em que as variáveis presentes no bloco 1 são consideradas distais (no presente estudo, considera-se que as características socioeconômicas e demográficas dos policiais exercem um efeito sobre os sucessivos níveis) na cadeia hierárquica do sofrimento psíquico; os blocos 2 e 3 são intermediários (a qualidade de vida e as condições de saúde dos policiais sofrem influência do nível anterior e têm efeito sobre o nível proximal); e o quarto bloco é o proximal (as condições de trabalho exercem influência direta sobre o desfecho).

A entrada das variáveis no modelo seguiu a orientação estabelecida pelos níveis hierárquicos, ou seja, o conjunto de variáveis que compõem o nível distal foi o primeiro a ser inserido no ajuste do modelo, seguindo até as variáveis do nível proximal. Cada variável estatisticamente significativa, de um nível hierárquico, permaneceu e foi analisada conjuntamente com o bloco subsequente. O critério de seleção de cada uma foi baseado no teste de Wald 19. Em todos os testes, o nível de significância adotado foi de $5 \%$.

Devido à natureza complexa do desenho da amostra, tanto nos testes de associação quanto nos ajustes dos modelos, foi incorporado o plano amostral para a correção das estimativas pontuais e da variância.

As análises estatísticas foram feitas nos programas SPSS (SPSS Inc., Chicago, Estados Unidos), versão 15.0, e SAS (SAS Inst., Cary, Estados Unidos), versão 9.1 .

O estudo foi aprovado pelo Comitê de Ética em pesquisa da Escola Nacional de Saúde Pública Sergio Arouca, Fundação Oswaldo Cruz, sob o parecer $n^{\circ}$. 27/03. Em todos os aspectos do trabalho, obedeceram-se as normas da Resolução 196/96, do Conselho Nacional de Saúde.

\section{Resultados}

A Tabela 1 mostra que a maioria dos policiais militares do Rio de Janeiro é do sexo masculino (96,3\%), está na faixa etária dos 31 aos 40 anos (43,1\%), 48,2\% são pardos, e $75,4 \%$ são casados. Quanto à situação religiosa, 52,9\% informaram que praticam algum tipo de religião. Observouse ainda que houve predomínio de policiais com escolaridade de 2 o grau incompleto e completo $(67,8 \%)$, embora $27,9 \%$ deles possuam ou este- jam cursando o nível superior ou pós-graduação. A renda familiar de $31,2 \%$ dos policiais militares encontra-se na faixa de $\mathrm{R} \$ 1.001,00$ a $\mathrm{R} \$$ $1.500,00$.

A análise verificou prevalência de sofrimento psíquico em 35,7\% (IC95\%: 32,9\%-38,6\%) dos policiais militares da cidade do Rio de Janeiro. Em relação ao tempo de trabalho, 13,2\% dos que estavam há dez anos na corporação apresentavam sofrimento psíquico, contra $24 \%$ dos que trabalhavam de $11-20$ anos e $16,2 \%$ dos que tinham mais de vinte anos na polícia (Tabela 1).

A fim de verificar independência, foi aplicado, inicialmente, o teste F ajustado. Na Tabela 2, podem ser visualizadas as variáveis testadas com respectivos OR (odds ratio) bruto e valor de $\mathrm{p}$.

No primeiro modelo, apenas a variável renda familiar do bloco perfil socioeconômico e demográfico foi incluída e mostrou-se significativa pelo teste de Wald ( $\mathrm{p}=0,0147$ ). Na construção do segundo modelo, ela foi acoplada ao grupo das variáveis de qualidade de vida que se mostraram associadas ao sofrimento psíquico $(\mathrm{p}<$ $0,05)$ na Tabela 2. Permaneceram significantes a propriedade da residência ( $p=0,032$ ), o grau de satisfação com a capacidade de reagir a situações difíceis $(p=0,001)$ e a satisfação com sua vida como um todo $(\mathrm{p}<0,001)$.

No terceiro modelo, foram incluídas as três variáveis relativas à qualidade de vida que se mostraram significantes $(p<0,05)$ e as relativas às condições de saúde. Em relação à qualidade de vida, permaneceram significativas a satisfação com a capacidade de reagir a situações difíceis ( $\mathrm{p}=0,004)$ e a satisfação com sua vida como um todo $(\mathrm{p}<0,001)$. Quanto às condições de saúde, houve significância para: prática de atividades físicas ( $p=0,008$ ), problemas no sistema digestivo ( $p=0,034)$, problemas nos músculos, ossos e pele $(\mathrm{p}=0,004)$, problemas do sistema nervoso ( $p<0,001)$ e problemas de visão, audição e fala ( $\mathrm{p}=0,010)$. Na última etapa (modelo 4 ), foram incluídas as variáveis referentes às condições de trabalho, dentre as quais, mantiveram-se significativas: todas as do modelo anterior, exceto a prática de atividades físicas $(\mathrm{p}=0,059)$ mais as variáveis relativas a trabalhar além do horário ( $\mathrm{p}=0,0201)$, estresse no trabalho $(\mathrm{p}<0,001)$ e vitimização ( $\mathrm{p}=0,047)$ (Tabela 3 ).

Ao observar o modelo logístico final (Tabela 4), conclui-se que existe mais chance de desenvolvimento de sofrimento psíquico entre os policiais que:

Do ponto de vista da qualidade de vida, não estão satisfeitos com sua capacidade de reagir a situações difíceis (1,998 vez mais que aqueles que estão muito satisfeitos ou satisfeitos); não estão satisfeitos com sua vida como um todo $(2,324$ 
Prevalência de sofrimento psíquico e perfil socioeconômico e demográfico dos policiais militares da cidade do Rio de Janeiro, Brasil, 2007.

\begin{tabular}{|c|c|}
\hline Variáveis & $\%$ (IC95\%) \\
\hline \multicolumn{2}{|l|}{ Sexo } \\
\hline Feminino & $3,7(2,7-5,0)$ \\
\hline Masculino & $96,3(95,0-97,3)$ \\
\hline \multicolumn{2}{|l|}{ Idade (anos) } \\
\hline Até 30 & $32,0(29,7-34,5)$ \\
\hline $31-40$ & $43,1(40,1-46,0)$ \\
\hline 41 ou mais & $24,9(23,1-26,9)$ \\
\hline \multicolumn{2}{|l|}{ Cor } \\
\hline Branca & $40,4(37,4-43,3)$ \\
\hline Preta & $9,8(8,1-11,7)$ \\
\hline Parda & $48,2(45,2-51,2)$ \\
\hline Amarela/Indígena & $1,7(1,0-2,7)$ \\
\hline \multicolumn{2}{|l|}{ Situação conjugal } \\
\hline Solteiro & $17,6(15,5-19,9)$ \\
\hline Casado & $75,4(72,7-77,8)$ \\
\hline Viúvo/Separado & $7,0(5,6-8,7)$ \\
\hline \multicolumn{2}{|l|}{ Prática de religião } \\
\hline Sim, frequentemente & $24,7(22,1-27,3)$ \\
\hline Sim, às vezes & $52,9(49,9-55,9)$ \\
\hline Não & $22,5(20,1-25,1)$ \\
\hline \multicolumn{2}{|l|}{ Escolaridade } \\
\hline 1ㅇ grau incompleto/completo & $4,2(3,2-5,6)$ \\
\hline 2o grau incompleto/completo & $67,8(65,1-70,4)$ \\
\hline Superior incompleto/completo/Pós-graduação & $27,9(25,5-30,5)$ \\
\hline \multicolumn{2}{|l|}{ Renda familiar (Reais) } \\
\hline Até 500 & $2,2(1,5-3,3)$ \\
\hline $501-1.000$ & $23,7(21,5-26,1)$ \\
\hline $1.001-1.500$ & $31,2(28,5-34,0)$ \\
\hline $1.501-2.500$ & $28,8(26,2-31,5)$ \\
\hline $2.501-4.000$ & $9,4(7,8-11,3)$ \\
\hline Mais de 4.000 & $4,7(3,7-5,9)$ \\
\hline \multicolumn{2}{|l|}{ Sofrimento psíquico } \\
\hline Presença & $35,7(32,9-38,6)$ \\
\hline Ausência & $64,3(61,4-67,1)$ \\
\hline
\end{tabular}

IC95\%: intervalo de $95 \%$ de confiança.

vezes mais que a chance daqueles que estão muito satisfeitos ou satisfeitos).

Em relação à saúde, têm problemas de sistema digestivo; musculares, ósseos e dermatológicos; do sistema nervoso, além dos problemas de visão, audição e fala, os quais estão diretamente correlacionados com a presença de sofrimento psíquico.

Em termos de condições de trabalho, desenvolvem poucas vezes atividades além do horário $(\mathrm{OR}=2,689)$; frequentemente apresentam es- tresse $(\mathrm{OR}=3,784$ vezes maior em relação àqueles que nunca ou quase nunca tiveram estresse no trabalho) e que sofreram alguma vitimização $(\mathrm{OR}=1,578)$.

\section{Discussão}

Observou-se, neste estudo, que fatores como capacidade de reagir a situações difíceis, grau de satisfação com a vida, comprometimento das 
Tabela 2

Odds ratio (OR) e teste entre variáveis independentes e sofrimento psíquico.

\begin{tabular}{|c|c|c|}
\hline Bloco/Variáveis & OR & Valor de $p$ \\
\hline \multicolumn{3}{|l|}{ Perfil } \\
\hline \multicolumn{3}{|l|}{ Idade (anos) } \\
\hline Até 30 & 1,04 & 0,748 \\
\hline $31-40$ & 0,93 & \\
\hline 41 ou mais & 1,00 & \\
\hline \multicolumn{3}{|l|}{ Sexo } \\
\hline Masculino & 1,19 & 0,635 \\
\hline Feminino & 1,00 & \\
\hline \multicolumn{3}{|l|}{ Cor } \\
\hline Amarela/Indígena & 1,15 & 0,126 \\
\hline Parda & 0,80 & \\
\hline Preta & 0,61 & \\
\hline Branca & 1,00 & \\
\hline \multicolumn{3}{|l|}{ Situação conjugal } \\
\hline Solteiro & 0,87 & 0,696 \\
\hline Viúvo/Separado & 1,01 & \\
\hline Casado & 1,00 & \\
\hline \multicolumn{3}{|l|}{ Tem filhos } \\
\hline Sim & 1,03 & 0,840 \\
\hline Não & 1,00 & \\
\hline \multicolumn{3}{|l|}{ Prática de religião } \\
\hline Não & 1,02 & 0,983 \\
\hline Sim, às vezes & 0,99 & \\
\hline Sim, frequentemente & 1,00 & \\
\hline \multicolumn{3}{|l|}{ Escolaridade } \\
\hline 1ㅇ grau incompleto/completo & 1,16 & 0,607 \\
\hline 2o grau incompleto/completo & 0,90 & \\
\hline Superior incompleto/completo/Pós-graduação & 1,00 & \\
\hline \multicolumn{3}{|l|}{ Renda familiar (Reais) } \\
\hline Até 500 & 6,20 & 0,011 \\
\hline $501-1.000$ & 2,89 & \\
\hline $1.001-1.500$ & 2,22 & \\
\hline $1.501-2.500$ & 2,30 & \\
\hline $2.501-4.000$ & 2,01 & \\
\hline Mais de 4.000 & 1,00 & \\
\hline \multicolumn{3}{|l|}{ Qualidade de vida } \\
\hline \multicolumn{3}{|l|}{ Mora em casa própria } \\
\hline Outra & 1,31 & 0,010 \\
\hline De favor & 1,85 & \\
\hline Alugada & 1,18 & \\
\hline Própria financiada & 0,87 & \\
\hline Própria quitada & 1,00 & \\
\hline \multicolumn{3}{|l|}{$\begin{array}{l}\text { Grau de satisfação com sua capacidade de reagir a situações } \\
\text { difíceis }\end{array}$} \\
\hline Nem satisfeito, nem insatisfeito/Insatisfeito/Muito insatisfeito & 2,98 & 0,000 \\
\hline Muito satisfeito/Satisfeito & 1,00 & \\
\hline
\end{tabular}

(continua) 
Tabela 2 (continuação)

\begin{tabular}{|c|c|c|}
\hline Bloco/Variáveis & OR & Valor de $p$ \\
\hline \multicolumn{3}{|l|}{ Qualidade de vida } \\
\hline \multicolumn{3}{|l|}{ Grau de satisfação com sua vida como um todo } \\
\hline Nem satisfeito, nem insatisfeito/Insatisfeito/Muito insatisfeito & 3,65 & 0,000 \\
\hline Muito satisfeito/Satisfeito & 1,00 & \\
\hline \multicolumn{3}{|l|}{ Apoio emocional } \\
\hline Baixo & 2,61 & 0,000 \\
\hline Médio & 1,58 & \\
\hline Alto & 1,00 & \\
\hline \multicolumn{3}{|l|}{ Apoio de informação } \\
\hline Baixo & 2,61 & 0,000 \\
\hline Médio & 1,49 & \\
\hline Alto & 1,00 & \\
\hline \multicolumn{3}{|l|}{ Interação positiva } \\
\hline Baixa & 2,13 & 0,000 \\
\hline Média & 1,00 & \\
\hline \multicolumn{3}{|l|}{ Apoio material } \\
\hline Baixo & 1,81 & 0,000 \\
\hline Médio & 1,00 & \\
\hline \multicolumn{3}{|l|}{ Apoio afetivo } \\
\hline Baixo & 2,22 & 0,000 \\
\hline Médio & 1,00 & \\
\hline \multicolumn{3}{|l|}{ Condições de saúde } \\
\hline \multicolumn{3}{|l|}{ Prática de atividades físicas } \\
\hline Não pratico & 3,77 & 0,000 \\
\hline Poucas vezes por ano & 2,43 & \\
\hline 2-3 vezes por mês & 1,93 & \\
\hline 1 vez por semana & 1,93 & \\
\hline $2-3$ vezes por semana & 1,09 & \\
\hline 4 ou + vezes por semana & 1,00 & \\
\hline \multicolumn{3}{|l|}{ Colesterol } \\
\hline Sim & 1,94 & 0,000 \\
\hline Não & 1,00 & \\
\hline \multicolumn{3}{|l|}{ Lesões permanentes causada pelo trabalho } \\
\hline Sim & 1,13 & 0,766 \\
\hline Não & 1,00 & \\
\hline \multicolumn{3}{|l|}{ Problemas no aparelho respiratório } \\
\hline Sim & 2,35 & 0,000 \\
\hline Não & 1,00 & \\
\hline \multicolumn{3}{|l|}{ Problemas no coração e aparelho circulatório } \\
\hline Sim & 2,70 & 0,000 \\
\hline Não & 1,00 & \\
\hline \multicolumn{3}{|l|}{ Problemas no sistema digestivo } \\
\hline Sim & 3,26 & 0,000 \\
\hline Não & 1,00 & \\
\hline \multicolumn{3}{|l|}{ Problemas nos músculos, ossos e pele } \\
\hline Sim & 3,48 & 0,000 \\
\hline Não & 1,00 & \\
\hline \multicolumn{3}{|l|}{ Problemas glandulares } \\
\hline Sim & 2,92 & 0,000 \\
\hline Não & 1,00 & \\
\hline
\end{tabular}

(continuação) 
Tabela 2 (continuação)

\begin{tabular}{|c|c|c|}
\hline Bloco/Variáveis & OR & Valor de $p$ \\
\hline \multicolumn{3}{|l|}{ Condições de saúde } \\
\hline \multicolumn{3}{|c|}{ Problemas do sistema nervoso } \\
\hline Sim & 7,26 & 0,000 \\
\hline Não & 1,00 & \\
\hline \multicolumn{3}{|c|}{ Problemas no aparelho urinário } \\
\hline Sim & 2,52 & 0,000 \\
\hline Não & 1,00 & \\
\hline \multicolumn{3}{|l|}{ Doenças transmissíveis } \\
\hline Sim & 3,12 & 0,000 \\
\hline Não & 1,00 & \\
\hline \multicolumn{3}{|c|}{ Problemas de visão, audição e fala } \\
\hline Sim & 2,72 & 0,000 \\
\hline Não & 1,00 & \\
\hline \multicolumn{3}{|l|}{ Consumo de substâncias } \\
\hline $\operatorname{Sim}$ & 1,68 & 0,001 \\
\hline Não & 1,00 & \\
\hline \multicolumn{3}{|l|}{ Condições de trabalho } \\
\hline \multicolumn{3}{|l|}{ Tempo de serviço (anos) } \\
\hline 26 ou mais & 0,29 & 0,037 \\
\hline $21-25$ & 1,15 & \\
\hline $16-20$ & 1,19 & \\
\hline $11-15$ & 1,80 & \\
\hline $16-10$ & 1,20 & \\
\hline Até 5 & 1,00 & \\
\hline \multicolumn{3}{|c|}{ Após entrar na polícia, sua vida (situação da vida após entrar } \\
\hline \multicolumn{3}{|l|}{ na polícia) } \\
\hline Piorou & 3,10 & 0,000 \\
\hline Continua igual & 1,54 & \\
\hline Melhorou & 1,00 & \\
\hline \multicolumn{3}{|c|}{ O trabalho que exerce é aquele para o qual treinou } \\
\hline Não & 1,65 & 0,000 \\
\hline $\operatorname{Sim}$ & 1,00 & \\
\hline \multicolumn{3}{|l|}{ Trabalhou além do horário } \\
\hline Sim, muitas vezes & 2,46 & 0,000 \\
\hline Sim, algumas vezes & 1,31 & \\
\hline Sim, poucas vezes & 2,12 & \\
\hline Não & 1,00 & \\
\hline \multicolumn{3}{|c|}{ Exerce outra atividade fora da polícia } \\
\hline Sim & 1,64 & 0,000 \\
\hline Não & 1,00 & \\
\hline \multicolumn{3}{|c|}{ Exerce atividade policial onde mora } \\
\hline $\operatorname{Sim}$ & 0,99 & 0,963 \\
\hline Não & 1,00 & \\
\hline \multicolumn{3}{|l|}{ Estresse no trabalho } \\
\hline Frequentemente & 3,07 & 0,000 \\
\hline Às vezes & 0,90 & \\
\hline Raramente & 0,44 & \\
\hline Nunca ou quase nunca & 1,00 & \\
\hline
\end{tabular}

(continua) 
Tabela 2 (continuação)

\begin{tabular}{lcc}
\hline Bloco/Variáveis & OR & Valor de $\mathbf{p}$ \\
\hline Condições de trabalho & & \\
$\quad$ No trabalho, as pessoas se relacionam bem umas com & & \\
as outras & 4,25 & $\mathbf{0}, 000$ \\
$\quad$ Discordo totalmente & 2,81 & \\
Discordo mais do que concordo & 1,53 & \\
Concordo mais do que discordo & 1,00 & \\
Concordo totalmente & & 0,428 \\
Percepção de risco & 1,31 & \\
Presença & 1,00 & 0,000 \\
Ausência & & \\
Vitimização & 2,55 & 1,00 \\
Presença & & \\
Ausência & & \\
&
\end{tabular}

Tabela 3

Variáveis associadas ao sofrimento psíquico de policiais militares da cidade do Rio de Janeiro, Brasil, segundo modelo hierarquizado, 2007.

\begin{tabular}{|c|c|c|c|c|c|c|c|c|}
\hline \multirow[t]{2}{*}{ Nível/Variável/Categorias } & \multicolumn{2}{|c|}{ Modelo 1} & \multicolumn{2}{|c|}{ Modelo 2} & \multicolumn{2}{|c|}{ Modelo 3} & \multicolumn{2}{|c|}{ Modelo 4} \\
\hline & OR & IC95\% & OR & IC95\% & OR & IC95\% & OR & IC95\% \\
\hline \multicolumn{9}{|l|}{ Perfil } \\
\hline \multicolumn{9}{|l|}{ Renda familiar (Reais) } \\
\hline Até 500 & 6,198 & $2,083-18,443$ & 3,333 & $1,017-10,92$ & & & & \\
\hline $501-1.000$ & 2,888 & $1,330-6,271$ & 1,582 & $0,694-3,604$ & & & & \\
\hline $1.001-1.500$ & 2,217 & $1,027-4,789$ & 1,402 & $0,621-3,166$ & & & & \\
\hline $1.501-2.500$ & 2,303 & $1,063-4,990$ & 1,997 & $0,897-4,446$ & & & & \\
\hline $2.501-4.000$ & 2,008 & $0,855-4,714$ & 1,972 & $0,806-4,824$ & & & & \\
\hline Mais de 4.000 & 1,000 & & 1,000 & & & & & \\
\hline \multicolumn{9}{|l|}{ Qualidade de vida } \\
\hline \multicolumn{9}{|l|}{ Morar em casa própria } \\
\hline Outra & & & 1,255 & $0,732-2,152$ & 1,149 & $0,638-2,069$ & & \\
\hline De favor & & & 1,646 & $1,052-2,576$ & 1,719 & $1,039-2,844$ & & \\
\hline Alugada & & & 0,957 & $0,646-1,418$ & 1,018 & $0,662-1,567$ & & \\
\hline Própria financiada & & & 0,678 & $0,426-1,081$ & 0,880 & $0,531-1,457$ & & \\
\hline Própria quitada & & & 1,000 & & 1,000 & & & \\
\hline \multicolumn{9}{|l|}{ Grau de satisfação com } \\
\hline \multirow{2}{*}{\multicolumn{9}{|c|}{$\begin{array}{l}\text { sua capacidade de reagir a } \\
\text { situações difíceis }\end{array}$}} \\
\hline & & & & & & & & \\
\hline Nem satisfeito, nem & & & 1,820 & $1,262-2,626$ & 1,898 & $1,226-2,938$ & 2,443 & $1,511-3,951$ \\
\hline \multicolumn{9}{|l|}{ insatisfeito/Insatisfeito/ } \\
\hline \multicolumn{9}{|l|}{ Muito insatisfeito } \\
\hline Muito satisfeito/ & & & 1,000 & & 1,000 & & 1,000 & \\
\hline Satisfeito & & & & & & & & \\
\hline
\end{tabular}

(continua) 
Tabela 3 (continuação)

\begin{tabular}{|c|c|c|c|c|c|c|c|c|}
\hline \multirow[t]{2}{*}{ Nível/Variável/Categorias } & \multicolumn{2}{|c|}{ Modelo 1} & \multicolumn{2}{|c|}{ Modelo 2} & \multicolumn{2}{|c|}{ Modelo 3} & \multicolumn{2}{|c|}{ Modelo 4} \\
\hline & OR & IC95\% & OR & IC95\% & OR & IC95\% & OR & IC95\% \\
\hline \multicolumn{9}{|l|}{ Qualidade de vida } \\
\hline \multicolumn{9}{|l|}{ Grau de satisfação com } \\
\hline \multicolumn{9}{|l|}{ sua vida como um todo } \\
\hline Nem satisfeito, nem & & & 2,494 & $1,801-3,456$ & 2,254 & $1,556-3,265$ & 2,091 & $1,411-3,098$ \\
\hline \multicolumn{9}{|l|}{ insatisfeito/Insatisfeito/ } \\
\hline \multicolumn{9}{|l|}{ Muito insatisfeito } \\
\hline Muito satisfeito/ & & & 1,000 & & 1,000 & & 1,000 & \\
\hline \multicolumn{9}{|l|}{ Satisfeito } \\
\hline \multicolumn{9}{|l|}{ Apoio emocional } \\
\hline Baixo & & & 1,429 & $0,703-2,905$ & & & & \\
\hline Médio & & & 1,478 & $0,856-2,551$ & & & & \\
\hline Alto & & & 1,000 & & & & & \\
\hline \multicolumn{9}{|l|}{ Apoio de informação } \\
\hline Baixo & & & 1,045 & $0,519-2,104$ & & & & \\
\hline Médio & & & 0,875 & $0,508-1,507$ & & & & \\
\hline Alto & & & 1,000 & & & & & \\
\hline \multicolumn{9}{|l|}{ Interação positiva } \\
\hline Baixa & & & 1,074 & $0,684-1,686$ & & & & \\
\hline Média & & & 1,000 & & & & & \\
\hline \multicolumn{9}{|l|}{ Apoio material } \\
\hline Baixo & & & 1,069 & $0,725-1,576$ & & & & \\
\hline Médio & & & 1,000 & & & & & \\
\hline \multicolumn{9}{|l|}{ Apoio afetivo } \\
\hline Baixo & & & 1,314 & $0,845-2,043$ & & & & \\
\hline Médio & & & 1,000 & & & & & \\
\hline \multicolumn{9}{|l|}{ Condições de saúde } \\
\hline \multicolumn{9}{|l|}{ Prática de atividades físicas } \\
\hline Não pratica & & & & & 2,752 & $1,464-5,173$ & 2,254 & $1,130-4,496$ \\
\hline Poucas vezes por ano & & & & & 2,129 & $1,127-4,023$ & 1,646 & $0,790-3,430$ \\
\hline 2-3 vezes por mês & & & & & 1,449 & $0,673-3,121$ & 1,309 & $0,574-2,984$ \\
\hline 1 vez por semana & & & & & 1,726 & $0,919-3,241$ & 1,571 & $0,778-3,171$ \\
\hline $2-3$ vezes por semana & & & & & 1,197 & $0,599-2,391$ & 0,993 & $0,460-2,144$ \\
\hline 4 ou + vezes por & & & & & 1,000 & & 1,000 & \\
\hline \multicolumn{9}{|l|}{ semana } \\
\hline Colesterol alto & & & & & & & & \\
\hline Sim & & & & & 1,345 & $0,899-2,014$ & & \\
\hline Não & & & & & 1,000 & & & \\
\hline Problemas no aparelho & & & & & & & & \\
\hline respiratório & & & & & & & & \\
\hline Sim & & & & & 1,403 & $0,981-2,006$ & & \\
\hline Não & & & & & 1,000 & & & \\
\hline Problemas no coração e & & & & & & & & \\
\hline aparelho circulatório & & & & & & & & \\
\hline $\operatorname{Sim}$ & & & & & 1,111 & $0,711-1,735$ & & \\
\hline Não & & & & & 1,000 & & & \\
\hline Problemas no sistema & & & & & & & & \\
\hline digestivo & & & & & & & & \\
\hline Sim & & & & & 1,493 & $1,031-2,163$ & 1,579 & $1,076-2,318$ \\
\hline Não & & & & & 1,000 & & 1,000 & \\
\hline
\end{tabular}

(continua) 
Tabela 3 (continuação)

\begin{tabular}{|c|c|c|c|c|c|c|c|c|}
\hline \multirow[t]{2}{*}{ Nível/Variável/Categorias } & \multicolumn{2}{|c|}{ Modelo 1} & \multicolumn{2}{|c|}{ Modelo 2} & \multicolumn{2}{|c|}{ Modelo 3} & \multicolumn{2}{|c|}{ Modelo 4} \\
\hline & OR & IC95\% & OR & IC95\% & OR & IC95\% & OR & IC95\% \\
\hline \multicolumn{9}{|l|}{ Condições de saúde } \\
\hline \multicolumn{9}{|l|}{$\begin{array}{l}\text { Problemas nos músculos, } \\
\text { nos ossos e pele }\end{array}$} \\
\hline Sim & & & & & 1,742 & $1,188-2,556$ & 1,600 & $1,058-2,420$ \\
\hline Não & & & & & 1,000 & & 1,000 & \\
\hline \multicolumn{9}{|l|}{ Problemas glandulares } \\
\hline Sim & & & & & 0,919 & $0,525-1,607$ & & \\
\hline Não & & & & & 1,000 & & & \\
\hline \multicolumn{9}{|l|}{ Problemas do sistema } \\
\hline \multicolumn{9}{|l|}{ nervoso } \\
\hline Sim & & & & & 4,405 & $3,129-6,203$ & 4,175 & $2,911-5,988$ \\
\hline Não & & & & & 1,000 & & 1,000 & \\
\hline \multicolumn{9}{|l|}{ Problemas no aparelho } \\
\hline \multicolumn{9}{|l|}{ urinário } \\
\hline Sim & & & & & 0,895 & $0,584-1,371$ & & \\
\hline Não & & & & & 1,000 & & & \\
\hline \multicolumn{9}{|l|}{ Doenças transmissíveis } \\
\hline $\operatorname{Sim}$ & & & & & 1,225 & $0,780-1,926$ & & \\
\hline Não & & & & & 1,000 & & & \\
\hline \multirow{2}{*}{\multicolumn{9}{|c|}{$\begin{array}{l}\text { Problemas de visão, } \\
\text { audição e fala }\end{array}$}} \\
\hline & & & & & & & & \\
\hline Sim & & & & & 1,584 & $1,115-2,251$ & 0,553 & $0,376-0,813$ \\
\hline Não & & & & & 1,000 & & 1,000 & \\
\hline \multicolumn{9}{|l|}{ Consumo de substâncias } \\
\hline Sim & & & & & 1,171 & $0,815-1,682$ & & \\
\hline Não & & & & & 1,000 & & & \\
\hline \multicolumn{9}{|l|}{ Condições de trabalho } \\
\hline \multicolumn{9}{|l|}{ Tempo de serviço (anos) } \\
\hline 26 ou mais & & & & & & & 0,411 & $0,085-1,991$ \\
\hline $21-25$ & & & & & & & 1,060 & $0,605-1,856$ \\
\hline $16-20$ & & & & & & & 1,317 & $0,772-2,247$ \\
\hline $11-15$ & & & & & & & 1,676 & $0,785-3,579$ \\
\hline $6-10$ & & & & & & & 1,139 & $0,698-1,859$ \\
\hline Até 5 anos & & & & & & & 1,000 & \\
\hline \multicolumn{9}{|l|}{ Vida após entrar na polícia } \\
\hline Piorou & & & & & & & 1,247 & $0,773-2,012$ \\
\hline Continua igual & & & & & & & 1,163 & $0,760-1,780$ \\
\hline Melhorou & & & & & & & 1,000 & \\
\hline \multirow{2}{*}{\multicolumn{9}{|c|}{$\begin{array}{l}\text { Exercer trabalho para o } \\
\text { qual foi treinado }\end{array}$}} \\
\hline & & & & & & & & \\
\hline $\operatorname{Sim}$ & & & & & & & 1,000 & \\
\hline Não & & & & & & & 1,304 & $0,896-1,897$ \\
\hline \multicolumn{9}{|l|}{ Trabalhar além do horário } \\
\hline Sim, muitas vezes & & & & & & & 2,074 & $0,901-4,777$ \\
\hline Sim, algumas vezes & & & & & & & 1,763 & $0,714-4,354$ \\
\hline Sim, poucas vezes & & & & & & & 3,699 & $1,460-9,371$ \\
\hline Não & & & & & & & 1,000 & \\
\hline
\end{tabular}

(continua) 
Tabela 3 (continuação)

\begin{tabular}{|c|c|c|c|c|c|c|c|c|}
\hline \multirow[t]{2}{*}{ Nível/Variável/Categorias } & \multicolumn{2}{|c|}{ Modelo 1} & \multicolumn{2}{|c|}{ Modelo 2} & \multicolumn{2}{|c|}{ Modelo 3} & \multicolumn{2}{|c|}{ Modelo 4} \\
\hline & OR & IC95\% & OR & IC95\% & OR & IC95\% & OR & IC95\% \\
\hline \multicolumn{9}{|l|}{ Condições de trabalho } \\
\hline \multicolumn{9}{|l|}{ Exercer outra atividade } \\
\hline \multicolumn{9}{|l|}{ fora da polícia } \\
\hline Sim & & & & & & & 1,221 & $0,842-1,769$ \\
\hline Não & & & & & & & 1,000 & \\
\hline \multicolumn{9}{|l|}{ Estresse no trabalho } \\
\hline Frequentemente & & & & & & & 2,983 & $1,457-6,109$ \\
\hline Às vezes & & & & & & & 1,120 & $0,544-2,304$ \\
\hline Raramente & & & & & & & 0,474 & $0,160-1,404$ \\
\hline Nunca ou quase nunca & & & & & & & 1,000 & \\
\hline \multicolumn{9}{|l|}{ Há bom relacionamento } \\
\hline \multicolumn{9}{|l|}{ entre as pessoas no } \\
\hline \multicolumn{9}{|l|}{ trabalho } \\
\hline Discordo totalmente & & & & & & & 1,964 & $0,973-3,967$ \\
\hline Discordo mais do que & & & & & & & 1,379 & $0,753-2,526$ \\
\hline \multicolumn{9}{|l|}{ concordo } \\
\hline Concordo mais do que & & & & & & & 1,297 & $0,725-2,319$ \\
\hline \multicolumn{9}{|l|}{ discordo } \\
\hline Concordo totalmente & & & & & & & 1,000 & \\
\hline \multicolumn{9}{|l|}{ Vitimização } \\
\hline Presença & & & & & & & 1,477 & $1,005-2,171$ \\
\hline Ausência & & & & & & & 1,000 & \\
\hline
\end{tabular}

IC95\%: intervalo de 95\% de confiança; OR: odds ratio.

condições de saúde física e mental, trabalho além do horário, estresse nas atividades laborais e a vitimização influenciam o desenvolvimento de sofrimento psíquico entre os policiais militares.

Antes de discutir os achados, é preciso comentar algumas limitações deste trabalho. Uma delas é o fato de se tratar de um estudo transversal que não permite verificar a relação de temporalidade entre o fator e o desfecho, nem a medida direta do risco, o que implica em prejuízo às inferências causais. Em relação ao modelo de regressão logística aqui usado, é importante destacar que ele permite estimar o OR, que é uma medida útil para investigar associação das variáveis ao desfecho, mas não é ideal para aferir a razão de prevalência, comumente utilizada em estudos transversais, pois, quando existe uma alta prevalência do desfecho, o OR tende a superestimar a razão de prevalência.

É possível que alguns vieses de seleção e de informação possam ter ocorrido, tendo, em vista, que os respondentes foram os policiais que aceitaram participar do estudo em cada unidade selecionada. Do mesmo modo, algumas questões podem ter sido sub-representadas como, por exemplo, o trabalho externo e o uso de subs- tâncias ou super-representadas, como o apoio social que consideram ter, também implicando em viés de informação. As distintas estratégias de coleta também podem ter influenciado tanto na seleção dos sujeitos como nas informações fornecidas, afetando os efeitos das variáveis independentes no desfecho, ora superestimando-o, ora subestimando-o.

O primeiro ponto a ser mencionado é o fato de nenhuma variável do perfil socioeconômico e demográfico dos policiais ter permanecido no modelo como explicativa do sofrimento psíquico. Mesmo a renda perdeu sua significância ao se incluírem as variáveis de qualidade de vida, indicando que a insatisfação com a capacidade de reagir a situações difíceis, frequentes no exercício das atividades policiais, e a insatisfação com a vida como um todo explicam mais o sofrimento psíquico que as características de idade, sexo, cor, situação conjugal ou mesmo a renda. Essa última, sempre muito ressaltada em estudos qualitativos e quantitativos 21,22.

Para Lipp 23, alguns profissionais que lidam com tarefas perigosas, como os policiais, afirmam que certa dose de estresse é positiva e necessária para que o serviço seja feito. A essa condição, o 
Tabela 4

Modelo logístico final das variáveis associadas ao sofrimento psíquico de policiais militares da cidade do Rio de Janeiro, Brasil, 2007.

\begin{tabular}{|c|c|c|c|c|c|c|}
\hline Bloco/Variáveis/Estratos & Prevalência (\%) & OR bruto & $\mathrm{IC} 95 \%$ & OR ajustado & IC95\% & Valor de $p$ * \\
\hline \multicolumn{7}{|l|}{ Qualidade de vida } \\
\hline \multicolumn{7}{|l|}{ Grau de satisfação com } \\
\hline \multicolumn{7}{|l|}{ sua capacidade de reagir a } \\
\hline \multicolumn{7}{|l|}{ situações difíceis } \\
\hline Nem satisfeito, nem & 56,1 & 2,974 & $2,189-4,040$ & 1,998 & $1,511-3,951$ & 0,0014 \\
\hline \multicolumn{7}{|l|}{ insatisfeito/Insatisfeito/Muito } \\
\hline \multicolumn{7}{|l|}{ insatisfeito } \\
\hline Muito satisfeito/Satisfeito & 30,1 & & & & & \\
\hline \multicolumn{7}{|l|}{ Grau de satisfação com sua } \\
\hline \multicolumn{7}{|l|}{ vida como um todo } \\
\hline Nem satisfeito, nem & 56,2 & 3,647 & $2,777-4,791$ & 2,324 & $1,634-3,307$ & $<0,001$ \\
\hline \multicolumn{7}{|l|}{ insatisfeito/Insatisfeito/Muito } \\
\hline \multicolumn{7}{|l|}{ insatisfeito } \\
\hline Muito satisfeito/Satisfeito & 26,0 & & & & & \\
\hline \multicolumn{7}{|l|}{ Condições de saúde } \\
\hline \multicolumn{7}{|l|}{ Problemas no sistema digestivo } \\
\hline Sim & 54,9 & 3,255 & $2,473-4,285$ & 1,735 & $1,229-2,449$ & 0,0017 \\
\hline Não & 27,2 & & & & & \\
\hline \multicolumn{7}{|l|}{ Problemas nos músculos, ossos } \\
\hline \multicolumn{7}{|l|}{ e pele } \\
\hline Sim & 46,6 & 3,475 & $2,629-4,594$ & 1,637 & $1,122-2,388$ & 0,0105 \\
\hline Não & 20,0 & & & & & \\
\hline \multicolumn{7}{|l|}{ Problemas do sistema nervoso } \\
\hline Sim & 62,5 & 7,263 & $5,480-9,626$ & 3,897 & $2,782-5,459$ & $<0,0001$ \\
\hline Não & 18,6 & & & & & \\
\hline \multicolumn{7}{|l|}{ Problemas de visão, audição } \\
\hline \multicolumn{7}{|l|}{ e fala } \\
\hline $\operatorname{Sim}$ & 47,1 & 2,723 & $2,103-3,528$ & 2,689 & $1,177-6,145$ & 0,0005 \\
\hline Não & 24,7 & & & & & \\
\hline \multicolumn{7}{|l|}{ Condições de trabalho } \\
\hline \multicolumn{7}{|l|}{ Trabalho além do horário } \\
\hline Sim, muitas vezes & 41,2 & 2,462 & $1,404-4,316$ & 1,690 & $0,812-3,515$ & 0,0470 \\
\hline Sim, poucas vezes & 37,7 & 2,124 & $1,077-4,190$ & 2,689 & $1,177-6,145$ & \\
\hline Sim, algumas vezes & 27,1 & 1,309 & $0,718-2,388$ & 1,335 & $0,610-2,917$ & \\
\hline Não & 22,2 & & & & & \\
\hline \multicolumn{7}{|l|}{ Estresse no trabalho } \\
\hline Frequentemente & 51,2 & 3,069 & $1,646-5,725$ & 3,784 & $1,871-7,651$ & $<0,0001$ \\
\hline Às vezes & 23,5 & 0,899 & $0,472-1,711$ & 1,152 & $0,560-2,367$ & \\
\hline Raramente & 13,0 & 0,439 & $0,192-1,006$ & 0,627 & $0,239-1,646$ & \\
\hline Nunca ou quase nunca & 25,5 & & & & & \\
\hline \multicolumn{7}{|l|}{ Vitimização } \\
\hline Presença & 50,3 & 2,552 & $1,946-3,345$ & 1,578 & $1,122-2,220$ & 0,0088 \\
\hline Ausência & 28,4 & & & & & \\
\hline
\end{tabular}

IC95\%: intervalo de 95\% de confiança; OR: odds ratio.

* Teste de Wald global: $p<0,0001$. 
autor denomina eustresse. Seu oposto é o estresse negativo ou distresse, que acovarda, intimida e faz com a pessoa fuja das situações.

Segundo a Organização Mundial da Saúde 24, a qualidade de vida é uma noção que envolve elevado grau de subjetividade e se associa também a moradia, recreação, lazer e transporte 25 . A análise indicou que a insatisfação com vários aspectos da vida, e o trabalho é um deles, aumenta as chances de sofrimento psíquico dos policiais militares. É importante recordar que a expectativa de satisfação humana com as diferentes dimensões da vida no âmbito familiar, amoroso, ambiental, social, profissional e existencial está relacionada ao padrão de conforto e bem-estar atingido historicamente pela sociedade ${ }^{5}$. Sobre isso, Martin \& Stockler 26 pontuam que, quanto menor for a distância que separa as expectativas individuais da realidade, melhor é a qualidade de vida.

Uma das expressões da qualidade de vida é o apoio social, definido por Due et al. 17 como os recursos disponibilizados por outras pessoas a favor de alguém, em situações de necessidade e que podem atuar como mediador e possibilitar formas adequadas de lidar melhor com perdas e problemas do cotidiano. Para avaliar esse componente, é necessário considerar a percepção do indivíduo sobre o suporte que reconhece ter. Vários autores ressaltam que pessoas socialmente mais integradas apresentam menos doenças e melhor prognóstico quando acometidas por enfermidades 17,18. Entretanto, nesta análise, nenhuma das dimensões do apoio social se mostrou associada ao sofrimento psíquico.

Expostos a cargas horárias extensivas e intensas de trabalho e a situações estressantes, os policiais tendem a desenvolver problemas de saúde que se cronificam ao longo do tempo, se não recebem atenção especial 1. Como lembra Dejours 12, embora o sofrimento humano seja intrínseco aos processos de trabalho, é necessário compreender suas causas para agir sobre elas, modificando-as com vistas a tornar o processo laboral um fator de saúde e não de adoecimento.

Neste estudo, verificou-se que a presença de certos distúrbios dos sistemas nervoso e digestivo, problema muscular, ósseo e de pele, além de problemas de visão, audição e fala aumentam o risco de sofrimento psíquico. Esses achados são corroborados por pesquisas com policiais civis do Rio de Janeiro 5 e por estudos com os policiais militares de Minas Gerais 27 e de Pernambuco ${ }^{8}$.

A variabilidade de eventos, em termos de sua natureza, circunstância, horário, local da ocorrência, duração da intervenção, atores e risco envolvidos, atribui imprevisibilidade ao trabalho policial que demanda ações resolutivas, sem o comprometimento da sua integridade física ou de outrem ${ }^{28}$.

Embora o risco seja estruturante das condições laborais, ambientais e relacionais da profissão policial, devido à iminência de vitimização que enfrentam cotidianamente, frequentemente, ele se transforma em situação real de vitimização e se traduz em traumas, lesões ou mortes que ocorrem nos confrontos com a criminalidade e na manutenção da ordem. Os resultados desta pesquisa indicam que os policiais que sofreram vitimização, seja em seu horário de trabalho, no lazer ou mesmo no lar, apresentam maiores chances de desenvolver sofrimento psíquico. Há os que são resilientes e amam o risco, porém alguns desenvolvem uma série de sintomas, dentre os quais, um estado de alerta permanente, estágio anterior ao estresse cumulativo, especialmente quando não se sentem satisfeitos com o trabalho $1,28,29,30,31$.

Pesquisa realizada com policiais civis e militares do Estado do Rio de Janeiro sobre riscos percebidos e vitimização 32 revela o crescimento da vitimização, nas duas corporações, por lesões não fatais, tendo, como causas principais, os acidentes de trânsito e as agressões. O mesmo estudo chama atenção para os altos índices de vitimização dos policiais em seus períodos de folga. Investigação com policial militar e civil e guarda municipal do Rio de Janeiro mostrou a maior vitimização letal e não letal da primeira, em relação às outras duas corporações 33 .

Divergindo do que seria esperado, observouse maior risco de sofrimento psíquico entre os policiais que afirmaram trabalhar poucas vezes além do horário quando ajustados os OR. Isso, por um lado, pode indicar um efeito de adaptação com diminuição do risco entre aqueles que excedem a carga horária de trabalho muitas vezes. Por outro lado, o crescimento do risco, após ajuste do OR, entre os que trabalham poucas vezes além do horário, comparados aos que o fazem muitas vezes, parece indicar o efeito da interferência de outras variáveis como, por exemplo, tempo de serviço e natureza do trabalho, que mereceriam aprofundamento da análise. Em última instância, esse achado também pode ser um efeito de viés de informação.

\section{Considerações finais}

O presente estudo permitiu delinear aspectos relacionados ao sofrimento psíquico entre policiais militares da cidade do Rio de Janeiro. O modelo logístico elaborado demonstrou que fatores como capacidade de reagir a situações difíceis, grau de satisfação com a vida, comprometimento da 
saúde física e mental, carga excessiva de trabalho, exposição constante ao estresse e à vitimização influenciam sobremaneira o desenvolvimento de sofrimento psíquico nesse grupo de profissionais.

Tais constatações, no entanto, vão além do processo de trabalho em si: suscitam reflexões acerca dos salários, do processo de organização institucional, dos cuidados dispensados à saúde e das condições de vida pessoais e familiares desses servidores. Merecem atenção especial os policiais que, pelas suas características de personalidade e pelo excesso de exposição ao risco e à vitimização, desenvolvem sintomas agudos e crônicos de sofrimento e de estresse cumulativo.

Este estudo aponta para a necessidade de medidas concretas, dentre as quais, o desenvolvimento de espaços de escuta dos problemas que os policiais vivenciam no cotidiano e em momentos de grandes tensões, visando não apenas, mas também, ao melhor desempenho técnico e a maior qualidade de vida para eles e suas famílias.

Dentre os possíveis desdobramentos, entende-se que os dados poderiam ser modelados com o enfoque de análise multinível, utilizando-se as distintas unidades/batalhões como variáveis de segundo nível, sob a hipótese de que policiais tenderiam a sofrer riscos dependendo da unidade/batalhão em que estariam alocados, repercutindo, assim, no seu sofrimento psíquico. Ressalta-se ainda a importância da realização de pesquisas que possam respaldar ações transformadoras, o que redundará em favor da corporação, do próprio policial, mas, sobretudo, da sociedade que precisa contar com a efetividade de seus serviços.

\section{Resumo}

Nesse artigo, foram investigados fatores associados ao sofrimento psíquico dos policiais militares da cidade do Rio de Janeiro, Brasil, a partir de estudo transversal com 1.120 policiais, sendo caracterizados o perfil socioeconômico e demográfico, a qualidade de vida, as suas condições de saúde e de trabalho. Para mensurar o sofrimento psíquico, utilizou-se o Self-Reported Questionnaire. Na análise de associações, usou-se o modelo de regressão logística, considerando-se variáveis relacionadas ao sofrimento psíquico. Os resultados indicaram associação entre sofrimento psíquico e fatores como: capacidade de reagir a situações difíceis e grau de satisfação com a vida; problemas de saúde, sobretudo, digestivos, nervosos, musculares e ósseos; e condições adversas de trabalho, como carga excessiva, constante exposição ao estresse e à vitimização. Conclui-se apontando a necessidade de intervenções que visem à promoção da saúde desses profissionais, sobretudo da sua saúde mental.

Estresse Psicológico; Polícia; Saúde Mental

\section{Colaboradores}

E. R. Souza colaborou na concepção, análise e redação do artigo. M. C. S. Minayo colaborou na revisão e escrita final do artigo. J. G. Silva colaborou na análise dos dados e redação do artigo. T. O. Pires colaborou na análise estatística dos dados e redação do artigo.

\section{Referências}

1. Gershon R, Lin S, Si X. Work stress in aging Police officers. J Occup Environ Med 2002; 44:160-7.

2. Cooper CL, Cartwright S. Organizational management of stress and destructive emotions at work. In: Payne RL, Cooper CL, editors. Emotions at work: theory, research and applications for management. Chichester: John Wiley \& Sons; 2001. p. 269-80.

3. Paschoal T, Tamoyo A. Validação da escala de estresse no trabalho. Estud Psicol (Natal) 2004; 9:4552.

4. Kelley TM. Mental health and prospective police professionals. Policing: an International Journal of Police Strategies \& Management 2005; 28:6-29.

5. Minayo MCS, Souza ER. Missão investigar: entre o ideal e a realidade de ser policial. Rio de Janeiro: Editora Garamond; 2003.

6. Minayo MCS, Souza ER, Constantino P. Missão prevenir e proteger: condições de vida, trabalho e saúde dos policiais militares do Rio de Janeiro. Rio de Janeiro: Editora Fiocruz; 2008.

7. Souza ER, Franco LG, Meireles CC, Ferreira VT, Santos NC. Sofrimento psíquico entre policiais civis: uma análise sob a ótica de gênero. Cad Saúde Pública 2007; 23:105-14.

8. Ferreira DKS, Augusto LGS, Jacqueline M. Condições de trabalho e percepção da saúde de policiais militares. Cad Saúde Colet (Rio J.) 2008; 16:403-20. 
9. Silva MB, Vieira SB. O processo de trabalho do militar estadual e a saúde mental. Saúde Soc 2008; 17:161-70.

10 Wisner A. A inteligência no trabalho: textos selecionados de ergonomia. São Paulo: Fundacentro; 1994.

11. Dejours C, Abdoucheli E. Itinerário teórico de psicopatologia do trabalho. In: Dejours C, Abdoucheli E, Jayet C, organizadores. Psicodinâmica do trabalho: contribuições da escola dejouriana à análise da relação prazer, sofrimento e trabalho. São Paulo: Editora Atlas; 1994. p. 119-45.

12. Dejours C. A loucura do trabalho: estudo de psicopatologia do trabalho. São Paulo: Editora Cortez; 1992.

13. Pessoa DGC, Nascimento Silva PLD. Análise de dados amostrais complexos. São Paulo: Associação Brasileira de Estatística; 1998.

14. Harding TW, de Arango MV, Baltazar J, Climent CE, Ibrahim HH, Ladrido-Ignacio L, et al. Mental disorders and primary health care: a study of their frenquency and diagnosis in four developing countries. Psychol Med 1980; 10:231-41.

15. Chor D, Griep RH, Faerstein E. Medidas de rede e apoio social no estudo Pró-Saúde: pré-testes e estudo piloto. Cad Saúde Pública 2001; 17:887-96.

16. Mari JJ, Williams PA. A validity study of a psychiatric screening questionnaire (SRQ 20) in primary care in the city of São Paulo. Br J Psychiatry 1986; 148:23-6.

17. Due P, Holstein B, Lund R, Modvig J, Avlund K. Social relations: network, support and relational strain. Soc Sci Med 1999; 48:661-73.

18. Holahan CJ, Moos RH. Social support and psychological distress: a longitudinal analysis. J Abnorm Psychol 1981; 90:165-70.

19. Vermeulen M, Mustard C. Gender differences in job strain, social support at work, and psychological distress. J Occup Health Psychol 2000; 5: 428-40.

20. Victora CG, Huttly SR, Fuchs SC, Olinto MTA. The role of conceptual frameworks in epidemiological analysis: a hierarchical approach. Int J Epidemiol 1997; 26:224-6.

21. Souza AL. Salários dos policiais militares no Brasil. http://www.diariodeumpm.net/ (acessado em 18/ Abr/2011).
22. Brito AS, Souza L. Representações sociais de policiais civis sobre profissionalização. Sociologias 2004; 6:304-27.

23. Lipp MEN. Pesquisa sobre stress no Brasil. São Paulo: Papirus Editora; 1996.

24. The WHOQOL Group. The World Health Organization Quality of Life position paper from the World Health Organization. Soc Sci Med 1995; 41:1403-9.

25. Andrade ER, Souza ER, Minayo MCS. Intervenção visando a autoestima e qualidade de vida dos policiais civis do Rio de Janeiro. Ciênc Saúde Coletiva 2009; 14:275-85.

26. Martin A, Stockler M. Quality of life assessment in health care research and practice. Eval Health Prof 1998; 21:141-56.

27. Minayo MCS. Avaliação dos riscos ambientais e das condições de saúde dos policiais militares de Minas Gerais. Belo Horizonte: Secretaria de Estado de Defesa Social; 2011.

28. Rodrigues CV, Rachid A. Análise de eventos em criminalística: um estudo de caso. In: XXVI Encontro de Engenharia de Produção [CD-ROM]. Fortaleza: Associação Brasileira de Engenharia de Produção; 2006.

29. Patis K. Stress e isolamento social versus stress e atividade de resgate ao público. In: Anais do Congresso da Associação Nacional de Medicina do Trabalho. v. 5. Florianópolis: Associação Nacional de Medicina do Trabalho; 1987. p. 785-9.

30. Glina DMR, Rocha LE, Batista ML, Mendonça MGV. Saúde mental e trabalho: uma reflexão sobre o nexo com o trabalho e o diagnóstico, com base na prática. Cad Saúde Pública 2001; 17:607-16.

31. Cusatis Neto R, Lima LM. Nível de estresse na Polícia Militar. Fisioter Bras 2003; 4:108-16.

32. Minayo MCS, Souza ER, Constantino P. Riscos percebidos e vitimização de policiais civis e militares na (in)segurança pública. Cad Saúde Pública 2007; 23:2767-79.

33. Souza ER, Minayo MCS. Policial, risco como profissão: morbimortalidade vinculada ao trabalho. Ciênc Saúde Coletiva 2005; 10:917-28.

Recebido em 09/Nov/2011

Versão final reapresentada em 27/Mar/2012 Aprovado em 03/Abr/2012 